\title{
La indagación científica y la transmisión-recepción: una contrastación de modelos de enseñanza para el aprendizaje del concepto densidad
}

\author{
Scientific inquiry and transmission-reception: a test of teaching models \\ for learning the concept of density
}

\section{Investigação científica e transmissão-recepção: um teste de modelos de ensino para aprender o conceito de densidade}

\author{
Elvira Flórez-Nisperuza ${ }^{1}$ \\ Andrés Fernando De la Ossa Albis ${ }^{2}$
}

Recibido: agosto de 2017

Aceptado: noviembre de 2017

Para citar este artículo: Flórez-Nisperuza, E., y De la Ossa Albis, A. (2018). La indagación científica y la transmisión-recepción: una contrastación de modelos de enseñanza para el aprendizaje del concepto densidad. Revista Científica, 31(1), 55-67. Doi: https://doi.org/10.14483/23448350.12452

\section{Resumen}

La indagación científica ha sido centro de atención de múltiples investigaciones en las últimas décadas; algunas respaldan y otras desacreditan su efectividad en el aprendizaje en relación con modelos de enseñanza más tradicionales como el de transmisión-recepción, que, para este estudio, se integran a prácticas de laboratorio coherentes con las respectivas metodologías. Este estudio tiene como objetivo principal determinar cuál de estas metodologías de la enseñanza tiene mayor influencia en el aprendizaje del concepto de densidad como propiedad de la materia en los niveles de conocimiento, comprensión y aplicación después de la intervención de cada estrategia, así como la indagación en el grupo experimental y la transmisión-recepción en el grupo de control. Por otra parte, se busca caracterizar los niveles de indagación alcanzados por los equipos conformados para realizar las prácticas de laboratorio del grupo sometido a dicho estrategia y relacionarlo con el aprendizaje. Para ello, se acude al paradigma cuantitativo de investigación y a un diseño cuasi-experimental de tipo transversal con pre y postest. Los resultados muestran de forma contundente que la indagación científica presenta mejores resultados en el aprendizaje del concepto en cuestión que la metodología de contraste con promedios de $(10,59)$ y $(8,18)$ respectivamente, corroborado estadísticamente por una prueba t que muestra un nivel de significancia de 0,017 . Además, el equipo de laboratorio que presentó mejor nivel de indagación obtuvo también el mejor promedio en el postest; sin embargo, atípicamente el equipo con el desempeño más bajo en la indagación superó al resto de equipos en dicho promedio.

Palabras clave: prácticas de laboratorio, formulación de hipótesis, diseño de experimentos, investigación.

Universidad de Córdoba. Montería, Córdoba, Colombia. Contacto: epatriciaflorez@correo.unicordoba.edu.co Universidad de Córdoba. Montería, Córdoba, Colombia. Contacto: afernandodelaossa@correo.unicordoba.edu.co 


\section{Abstract}

The scientific inquiry has been the focus of multiples researches in the last decades, some support and others discredit its effectiveness in learning in relation to more traditional teaching models such as transmission-reception, that for this study, are integrated into laboratory practices consistent with the respective methodologies. The main objective of the research is to determine which of these teaching methodologies have the greatest influence in the learning of the concept of density as property of matter in the levels of knowledge, understanding, and implementation after the intervention of such strategy; inquiry in the experimental group and, transmission-reception in the control group, and, furthermore, characterize the levels of inquiry reached by the different teams formed to carry out the laboratory practices of the group subjected to said strategy and related it to learning. To achieve this is used the quantitative paradigm of investigation and a quasi-experimental design of transversal type with pre-and post-test. The results show conclusively that the scientific inquiry present best results in learning the concept in question, other than the methodology of contrast with an average of $(10,59)$ and $(8,18)$ respectively, statically corroborated by a test $T$ that shows a significant level of 0,017 . In addition, the laboratory team that presented the best level of inquiry also obtained the best average in the post-test, nevertheless, unlike the team with the lowest performance in the inquiry, exceeded the rest of the teams in said average.

Keywords: lab practices, hypothesis formulation, design of experiments, investigation.

\section{Resumo}

O inquérito científico tem sido o foco de pesquisas múltiplas nas últimas décadas, alguns apoios e outros desacreditam a sua eficácia na aprendizagem em relação a modelos de ensino mais tradicionais como a transmissão-recepção, que, para este estudo, são integrados em práticas laboratoriais consistentes com a respectivas metodologias. O principal objetivo da pesquisa é determinar quais dessas metodologias de ensino têm a maior influência na aprendizagem do conceito de densidade como propriedade da matéria nos níveis de conhecimento, compreensão e implementação após a intervenção de tal estratégia; inquérito no grupo experimental e transmissão-recepção no grupo de controle e, além disso, caracterizam os níveis de inquérito alcançados pelas diferentes equipas formadas para realizar as práticas laboratoriais do grupo sujeito à referida estratégia e relacionou-a à aprendizagem. Para conseguir isso é usado o paradigma quantitativo de investigação e um projeto quase experimental de tipo transversal com pré e pós-teste. Os resultados mostram de forma conclusiva que o inquérito científico apresenta os melhores resultados na aprendizagem do conceito em questão, além da metodologia de contraste com uma média de $(10,59)$ e $(8,18)$, respectivamente, corroborado estaticamente por um teste T que mostra um nível significativo de 0,017. Além disso, a equipe de laboratório que apresentou o melhor nível de pesquisa também obteve a melhor média no pós-teste, no entanto, ao contrário da equipe com o menor desempenho no inquérito, excedeu o resto das equipes na referida média.

Palavras-chaves: práticas laboratoriais, formulação de hipóteses, projeto de experiências, pesquisa.

\section{Introducción}

Existen numerosas investigaciones que han buscado establecer si la indagación científica es realmente la mejor forma de enseñar y aprender ciencia. Haciendo un breve recorrido por los estudios que se han dedicado a analizar la temática, puede notarse que la opinión al respecto está dividida, es decir, algunas la respaldan mientras otras la desacreditan. A favor de la indagación científica encontramos, por ejemplo, la opinión de Lederman, Lederman y Antink (2013), quienes afirman que la mejor forma de aprender ciencia es a través de la indagación. Se cree que los estudiantes aprenden mejor los conceptos científicos al hacer ciencia. En este mismo sentido, Romero-Ariza (2017) ilustra los resultados de una serie de investigaciones que demuestran los beneficios de la indagación científica en la enseñanza de las ciencias. Según este, las investigaciones en las que se emplean metodologías que promueven la capacidad de pensar y 
argumentar con base en evidencias representan un factor positivo aunque moderado en la comprensión de ideas científicas, en especial cuando lo que se busca es la produndización del tema.

Por otro lado, existen voces en contra de la implementación de la indagación en la escuela. Así, Sweller y Clark (2006) afirman que las actividades abiertas de indagación, de aprendizaje basado en problemas, por descubrimiento y de naturaleza constructivistas están irremediablemente condenadas al fracaso, pues desconocen la arquitectura cognitiva humana. Estos autores hacen énfasis en el papel fundamental de la guía y la instrucción, ya que de esta manera se refuerza la memoria de largo plazo, un aspecto fundamental dentro de la arquitectura cognitiva humana. Por su parte, Couso Lagarón (2014) señala que muchos trabajos de indagación se planten sin un objetivo conceptual y, en su lugar, lo único que pretenden es aprender a indagar, "iy encima de eso mal!". Según la investigadora, esto sucede porque se muestra el método científico de una forma muy simplificada que termina distorsionando la naturaleza de la ciencia. Asimismo, Martin Hasen (2002) afirma que no son pocas las actividades que se promocionan como indagatorias sin que realmente tengan las características propias de la indagación. Visto de este modo, los resultados negativos en el aprendizaje conceptual son más bien atribuibles a un mal enfoque de la indagación en las actividades académicas propuestas.

Teniendo en cuenta las posturas en favor y en contra de la indagación como modelo de enseñanza y aprendizaje, y que son numerosas las actividades de indagación descalificadas debido a una mala interpretación del término, se hace pertinente poner a prueba una actividad diseñada por los investigadores en este estudio y compararla con una metodología más bien heteroestructurante que haga énfasis en la instrucción.

\section{Metodología}

El estudio se alinea con el paradigma cuantitativo de investigación y es de tipo transversal, pues las medidas se realizaron en los mismos tiempos y en una sola oportunidad. El diseño corresponde a un cuasi-experimento con pretest, postest y grupo de control, ya que al tratarse de grupos intactos fue necesario saber qué tan similares son los grupos con el fin de disminuir el sesgo al comparar la efectividad de las metodologías puestas a prueba, En otras palabras, si el estímulo de la variable independiente es responsable o no de los eventuales efectos (positivos o negativos) sobre la variable dependiente (Hernández y Fernández, 2010). Los análisis estadísticos se realizaron con el software SPSS versión 23 y las gráficas con Excel. Es preciso decir que los modelos de enseñanza fueron integrados a prácticas de laboratorios coherentes con sus metodologías. Se trata de una práctica tipo investigación que partió de un problema conceptual (véase anexo B) y con el cual los propios estudiantes formularon sus hipótesis y diseñaron sus experimentos he implementada en el grupo experimental; mientras que al grupo de control se aplicó una práctica de laboratorio tipo "receta" o de comprobación teórica en la que el diseño experimental estaba pre-establecido en la guía de trabajo.

\section{Instrumentos}

Para establecer el tipo de aprendizaje, se aplicó un cuestionario de 20 preguntas cerradas antes y después de implementar las estrategias de enseñanza en cada uno de los grupos. Este ayudó a evaluar el aprendizaje de la densidad como propiedad de la materia en los niveles de conocimiento, comprensión y aplicación. El cuestionario fue validado por dos expertos y aplicado previamente en una prueba piloto con el que se le determinó una confiabilidad de homogeneidad interna de 0,72 con el modelo de Kuder-Richardson, donde 0,0 representa confiabilidad nula y 1,0 representa confiabilidad máxima (Ruiz Bolívar, 2008). Sin embargo, el instrumento cuenta con una confiabilidad alta por lo que se asume la medición de la variable en consideración. 
Al mismo tiempo, se aplicó un instrumento para caracterizar el nivel de indagación alcanzado por los seis equipos de laboratorio correspondientes al grupo experimental New Practical Test Assessment Inventory o NPTAI (Ferrés Gurt, Marbá Tallada y Neus, 2015), una escala ordinal que cuenta con unas categorías las cuales evalúan el desempeño de indagación, para ello fue modificado y adaptado a las condiciones académicas de la población y a los recursos disponibles en la institución para la realización de los trabajos prácticos, entre ellos: la escaza familiarización con la metodología de la indagación científica, la nula experiencia en trabajos prácticos de laboratorio en grados anteriores (inclusive en prácticas de laboratorio tipo "receta"), así como la disponibilidad de materiales, reactivos y aparatos de laboratorio de la institución. Por tal razón ha sido denominado nivel de indagación científica para principiantes (NdICpP) y validado por dos expertos. Las categorías tenidas consideradas para la caracterización fueron: $\mathrm{A}=$ formulación de hipótesis, $\mathrm{B}=$ diseño de experimentos, $\mathrm{C}=$ identificación de variables, $\mathrm{D}=$ recolección y procesamiento de datos, $\mathrm{E}=$ análisis de datos y obtención de conclusiones. Cada una de estas categorías (cinco en total) tiene un número determinado de rúbricas ordinales. Teniendo en cuenta que las categorías difieren en el número de rúbricas, se le asignó un valor de 5 puntos a cada una para igualar su aporte al NdICpP, de modo que la puntuación máxima del instrumento es de 25 puntos; el valor de cada rúbrica se determina con el cociente $5 / \mathrm{n}-1$, donde n representa el número de rúbricas de la categoría, se hace la corrección $n-1$ porque el instrumento contempla valoraciones de cero (0) (véase anexo A).

\section{Propuesta metodológica de la indagación científica}

Para comprobar que la indagación científica es una estrategia que produce mejores resultados en el aprendizaje conceptual en comparación con el modelo de transmisión-recepción, a continuación, se detalla las etapas y pasos seguidos durante su implementación.

El grupo experimental se organizó en seis equipos: cinco grupos de cinco estudiantes y un grupo de cuatro, a los cuales se les valoró en cada una de las categorías de indagación puestas a prueba en el instrumento NdICpP.

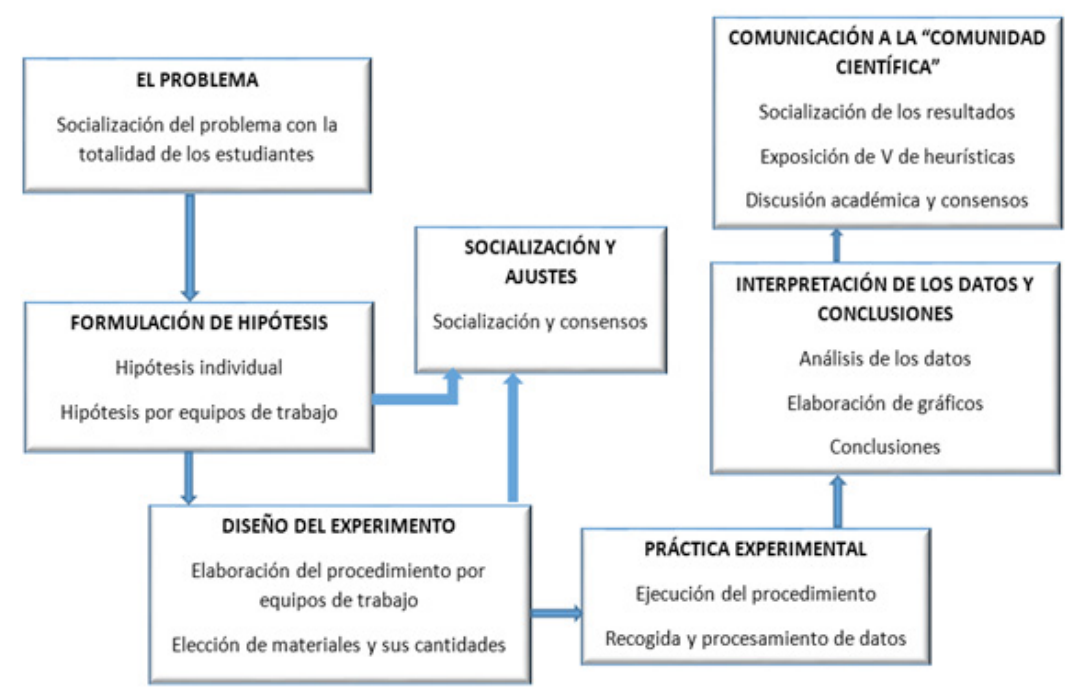

Figura 1. Etapas y procesos para la implementación de la estrategia basada en la indagación científica.

Fuente: adaptada del modelo de investigación propuesto por la Assessment of Performance Unit, citado en Caamaño (2003). 


\section{Población y muestra}

El estudio se realizó en una población de 1.364 estudiantes de la Institución Educativa la Ye del municipio de Sahagún, en el departamento de Córdoba. En específico, una muestra de 57 estudiantes con un promedio de 14 años correspondientes a los grados noveno $\mathrm{A}\left(9^{\circ} \mathrm{A}\right)$ y noveno $\mathrm{B}$ $\left(9^{\circ} \mathrm{B}\right)$ para los grupos de control y experimental respectivamente.

\section{Resultados y discusión}

En primer lugar, se analiza el pretest con el que se determinó el grado de equivalencia entre el grupo de control y experimental.

Los resultados evidencian medias y desviaciones estándar muy similares entre los grupos de comparación, por lo cual fue necesario corroborar estadísticamente la similitud de los grupos de la siguiente forma:

Se realizó la prueba de normalidad de la variable cuantitativa de calificación (puntos correctos de 20 posibles), atendiendo a los supuestos mostrados a continuación. Se acude a la prueba de Shapiro-Wilk por tratarse de muestras menores a 30 puntos.

- $\quad$ Si P-valor $>\alpha(0,05)$ Aceptar $\mathrm{H0}=$ se acepta que los datos provienen de una distribución normal

- Si P-valor $<\alpha(0,05)$ Aceptar $\mathrm{H} 1$ = se rechaza que los datos provienen de una distribución normal

De los resultados de la prueba puede notarse que la variable en cuestión en el grupo de experimental se comporta de forma normal, mientras que para el grupo de control no.

$$
\begin{aligned}
& P-\text { valor (experimental })=0,122>\alpha=0,05 \\
& P-\text { valor }(\text { control })=0,009<\alpha=0,05
\end{aligned}
$$

Dado que no se satisfacen varias de las condiciones de parametricidad, se aplica la prueba no paramétrica de $U$ de Mann de Whitney que compara las medianas de las muestras, no sin antes establecer el siguiente sistema de hipótesis:

Tabla 1. Resumen estadístico de los resultados del pretest.

\begin{tabular}{lllllll}
\multicolumn{1}{c}{ Grupo } & Media & $\mathbf{N}$ & $\begin{array}{c}\text { Desviación } \\
\text { estándar }\end{array}$ & Mínimo & Máximo & Varianza \\
\hline Experimental & 7,17 & 29 & 2,189 & 3 & 13 & 4,791 \\
\hline Control & 7,00 & 28 & 2,749 & 4 & 14 & 7,556 \\
\hline Total & 7,09 & 57 & 2,459 & 3 & 14 & 6,046 \\
\hline
\end{tabular}

Fuente: elaboración propia.

\begin{tabular}{|c|c|c|c|c|}
\hline \multirow{3}{*}{ Pretest } & \multirow{2}{*}{$\begin{array}{l}\text { Grupo } \\
\text { Experimental }\end{array}$} & \multicolumn{3}{|c|}{ Shapiro-Wilk } \\
\hline & & 943 & 29 &, 122 \\
\hline & Control & ,896 & 28 & ,009 \\
\hline
\end{tabular}

Tabla 2. Prueba de normalidad de Shapiro-Wilk para el pretest

Fuente: elaboración propia. 
- $\quad$ Si P- valor $>\alpha(0,05)$ Aceptar $\mathrm{HO}=$ los grupos no presentaron diferencias significativas

- $\quad$ Si P-valor $<\alpha(0,05)$ Aceptar $\mathrm{H} 1$ = los grupos presentan diferencias significativas

Tabla 3. Prueba de U de Mann-Whitney para probar diferencia o similitud de los grupos experimental y de control.

\begin{tabular}{ll} 
U de Mann-Whitney & 360,500 \\
\hline W de Wilcoxon & 766,500 \\
\hline$Z$ &,- 735 \\
\hline Sig. asintótica (bilateral) &, 463 \\
\hline
\end{tabular}

Fuente: elaboración propia.

La significancia (sig. asintótica bilateral) arrojada por la prueba es mayor que alfa $(\alpha)$, así que se acepta la hipótesis nula. Con ello se concluye que los grupos no presentan diferencias significativas.

Este resultado fue positivo para la investigación, pues no fue necesario realizar ningún emparejamiento de los grupos y se posibilitó continuar con el diseño cuasi-experimental planteado. De esta forma, las diferencias presentadas en los aprendizajes de los grupos son atribuibles con mayor probabilidad a las características de las metodologías.

$$
\mathrm{P}-\text { valor }=0,463>\alpha=0,05
$$

Ahora bien, con el análisis del postest se establece finalmente cuál metodología tuvo mayor influencia sobre el aprendizaje de la densidad. A continuación, se muestra el resumen estadístico de la segunda aplicación del test.

Al igual que en el pretest, se realizó una prueba de normalidad para decidir el estadístico apropiado para el análisis inferencial del postest. Por tanto, se aplicó la misma prueba para este fin.

En la tabla se aprecia que $\mathrm{P}$ - valor o la significancia (sig.) es mayor que el nivel alfa $(\alpha)$ para ambos grupos.

$$
\begin{aligned}
& P-\text { valor }(\text { control })=0,444>\alpha=0,05 \\
& P-\text { valor }(\text { experimental })=0,527>\alpha=0,05
\end{aligned}
$$

Con estos valores se acepta la hipótesis nula, es decir, se establece que la variable en tratamiento se comporta de forma normal en ambos casos. Una vez pasado este filtro, se determinó la igualdad de varianza con la prueba de Levene según el siguiente sistema de hipótesis.

Tabla 4. Resumen estadístico de los resultados del postest.

\begin{tabular}{llllll} 
& Grupo & $N$ & Media & $\begin{array}{l}\text { Desviación } \\
\text { estándar }\end{array}$ & $\begin{array}{l}\text { Media de error } \\
\text { estándar }\end{array}$ \\
\cline { 2 - 6 } Postest & Experimental & 29 & 10,59 & 3,541 &, 658 \\
\cline { 2 - 6 } & Control & 28 & 8,18 & 3,850 &, 727 \\
\hline
\end{tabular}

Fuente: elaboración propia.

Tabla 5. Prueba de normalidad de Shapiro-Wilk para el postest.

\begin{tabular}{lcccc}
\multirow{4}{*}{ Postest } & Grupo & \multicolumn{3}{c}{ Shapiro-Wilk } \\
\cline { 2 - 5 } & Experimental & Estadístico & Gl & Sig. \\
\cline { 2 - 5 } & Control &, 965 & 29 &, 444 \\
\cline { 2 - 5 } & & & 28 &, 527 \\
\hline
\end{tabular}

Fuente: elaboración propia. 
- $\mathrm{HO}=$ las varianzas son iguales si $\mathrm{P}$ - valor $>\alpha \mid \mathrm{fa}$ $(0,05)$

- $\mathrm{H} 1$ = si existe diferencias significativas entre las varianzas si $\mathrm{P}$ - valor $<\alpha$ lfa $(0,05)$

Tabla 6. Prueba de Levene para probar igualdad de varianzas de los resultados del postest en grupos de control y experimental.

\begin{tabular}{cccc} 
& & \multicolumn{2}{c}{$\begin{array}{c}\text { Prueba de Levene de } \\
\text { igualdad de varianzas }\end{array}$} \\
\cline { 3 - 4 } & $\mathrm{F}$ & Sig. \\
\hline \multirow{3}{*}{ Postest } & $\begin{array}{c}\text { Se asumen } \\
\text { varianzas iguales }\end{array}$ &, 099 &, 755 \\
\cline { 2 - 4 } & $\begin{array}{c}\text { No se asumen } \\
\text { varianzas iguales }\end{array}$ & \\
\hline
\end{tabular}

Fuente: elaboración propia.

Dadas las condiciones y el resultado de la prueba de Levene, se asumen varianzas iguales en la variable dependiente para los dos grupos, ya que el nivel de significancia (sig.) o $\mathrm{P}$ - valor es mayor que alfa $(\alpha)$.

$$
\mathrm{P}-\text { valor }=0,755>\alpha=0,05
$$

Con estos resultados se pudo confirmar el comportamiento normal de la variable, y además se asume igualdad de varianza. Ahora, conforme a los resultados obtenidos, se aplicó la prueba T para muestras independientes para la comparación de medias con el propósito de determinar si la diferencia presentada en las medias de los grupos de control y experimental son significativas; para esto se estableció que:
Si P- valor $>\alpha(0,05)$ Aceptar $=\mathrm{HO}$ : la indagación científica integrada a los trabajos prácticos de laboratorio no representa una metodología que permite el aprendizaje conceptual en mayor grado que los métodos de enseñanza de transmisión-recepción apoyado en el uso de prácticas de laboratorio tipo "receta".

Si P-valor $<\alpha(0,05)$ Aceptar H1 = la indagación científica integrada a los trabajos prácticos de laboratorio representa una metodología que permite el aprendizaje conceptual en mayor grado que los métodos de enseñanza de transmisión-recepción apoyado en el uso de prácticas de laboratorio tipo "receta".

Si se concentra la atención en la significancia expuesta en la tabla 7 y se confronta con el sistema de hipótesis que compara las estrategias de enseñanza, se evidencia que esta es menor que alfa $(\alpha)$ 0,05 . En este caso, los valores de significancia son iguales en ambas filas (columna 3 de la tabla 7), así que no hay que hacer una elección entre ellas.

En definitiva, el valor de la significancia es menor que el de alfa $(\alpha)$, por lo que se rechaza la hipótesis nula o, en su defecto, se acepta la hipótesis de investigación H1.

$$
P-\text { valor }=0,017<\alpha=0,05
$$

Se concluye entonces, a partir de la prueba estadística t student para muestras independientes, que en la estrategia fundamentada en la indagación científica integrada a una práctica de laboratorio de esta naturaleza se logró mejores aprendizajes conceptuales que la estrategia basada en el modelo de transmisión-recepción integrada a una práctica de laboratorio tipo "receta".

Tabla 7. Prueba t para muestras independientes del postest.

Prueba t para la igualdad de medias

\begin{tabular}{llccccc}
\hline \multirow{2}{*}{ T } & Gl & Sig. (bilateral) & $\begin{array}{c}\text { Diferencia de } \\
\text { medias }\end{array}$ & $\begin{array}{c}\text { Diferencia de } \\
\text { error estándar }\end{array}$ & & \multicolumn{2}{c}{$\begin{array}{c}95 \% \text { de intervalo de confianza de la } \\
\text { diferencia }\end{array}$} \\
\hline 2,459 & 55 &, 017 & 2,408 &, 979 & Inferior & Superior \\
\hline 2,455 & 54,232 &, 017 & 2,408 &, 985 & 4,370 \\
\hline
\end{tabular}

Fuente: elaboración propia. 


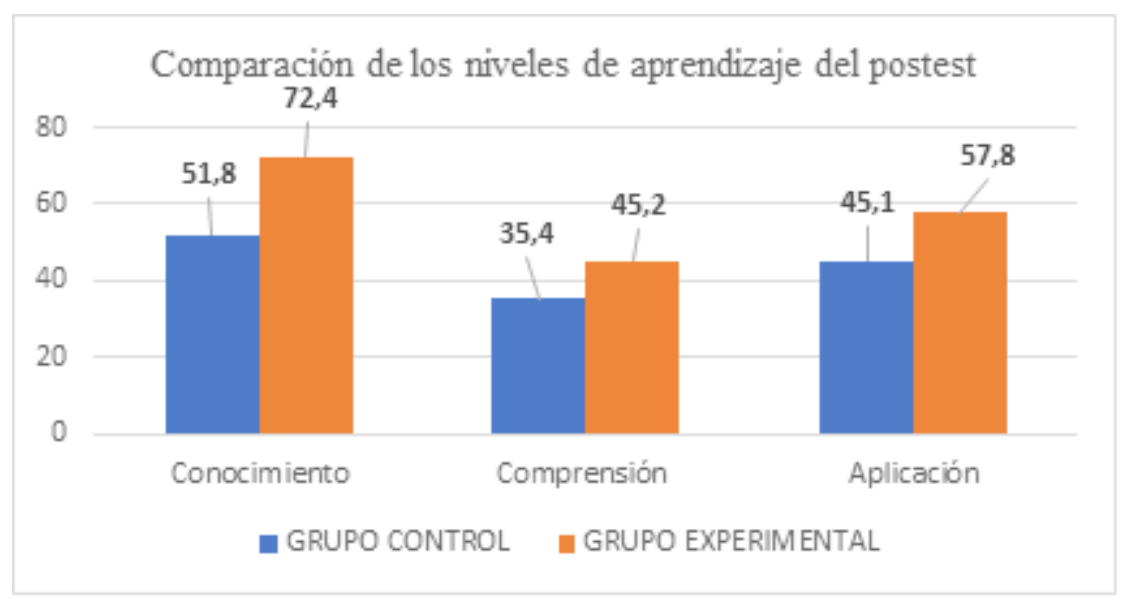

Figura 2. Comparación de los desempeños alcanzados en el aprendizaje en los niveles de conocimiento, comprensión y aplicación de los grupos experimental y de control en el postest.

Fuente: elaboración propia.

En relación con los tres niveles de aprendizaje conceptual (conocimiento, comprensión y aplicación), se observó que estos fueron superiores en el grupo experimental en cada caso. En especial, destacan la diferencia en el nivel de conocimiento (20,6 puntos porcentuales), seguido por el de aplicación (los cuales difieren en 12,7$)$ y, finalmente, el de comprensión (con una diferencia de 9,8). Desde esta perspectiva, queda respaldada aún más la hipótesis de investigación.

De forma análoga, se muestran los niveles de indagación alcanzados por los seis equipos de trabajo en el laboratorio, correspondiente al grupo experimental en cada una de las cinco categorías tenidas en cuenta.

La categoría en donde se logró un mayor desempeño por parte de los equipos de trabajo en el laboratorio es la formulación de hipótesis (categoría A), con un promedio de 3,5. Por el contrario, la categoría de indagación en la que mayor dificultad presentaron dichos equipos es: el análisis de datos y la obtención de conclusiones (categoría E), con un promedio de tan solo 0,3 ; seguida por el diseño de experimentos (categoría B), con un promedio de 1,4.

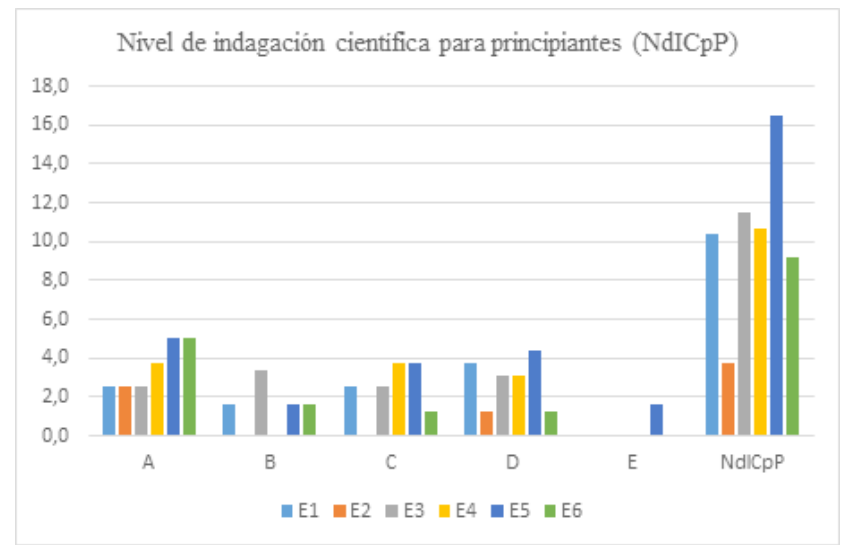

Figura 3. Niveles de indagación alcanzados por los seis equipos de laboratorio del grupo experimental.

Fuente: elaboración propia.

Según los reportes de investigaciones recientes, las competencias que representan mayor dificultad para los estudiantes son la formulación de hipótesis y el diseño del experimento (Ferrés Gurt, Marbá Tallada, y Neus, 2015; González Rodríguez y Crujeiras Pérez, 2016), pues lo regular es que ellos reciban las instrucciones listas en el procedimiento de una guía de laboratorio en lugar de elaborar el procedimiento. Esto se acentúa aún más ya que, como se mencionó antes, ni siquiera en prácticas tipo 
"receta" tienen experiencia. Sin embargo, la formulación de hipótesis en este estudio tuvo un comportamiento atípico si se compara con otros relacionados; tan es así que los equipos 5 y 6 lograron la puntuación máxima en esta categoría $(5,0)$. Lo anterior se puede entender desde la forma deductiva como se abordó la construcción de la hipótesis, más explícitamente a la disposición de unos referentes teóricos relacionados con el concepto que llevaron a la explicación del problema, para el caso de la densidad: las fuerzas de cohesión y su relación con la masa y el volumen ocupado de las sustancias consideradas en el problema, además del acompañamiento del docente a través de cuestionamientos que ayudaron en la elaboración de la conjetura. En general, el bagaje del maestro fue imprescindible en la realización de prácticas de indagación. Las pistas, las indicaciones, las preguntas abiertas y la activación del conocimiento teórico son recursos de apoyo sin los cuales sería muy difícil completar la tarea de forma autónoma (Crujeiras Pérez y Jiménez Aleixandre, 2015).

Finalmente, se comparó el NdlcpP alcanzado por los seis equipos de laboratorio con el promedio de calificación obtenida en el postest, teniendo en cuenta que la indagación se valoró de forma grupal.

Tabla 8. NdIcpP obtenida por los equipos y sus correspondientes promedios obtenidos en el postest.

\begin{tabular}{ccc}
\hline Equipos & NdICpP & $\begin{array}{c}\text { Promedio } \\
\text { calificación }\end{array}$ \\
\hline E1 & 10,4 & 9,6 \\
E2 & 3,8 & 10,2 \\
E3 & 11,5 & 10,0 \\
E4 & 10,6 & 9,5 \\
E5 & 16,5 & 14,2 \\
E6 & 9,2 & 9,8 \\
\hline
\end{tabular}

Fuente: elaboración propia.

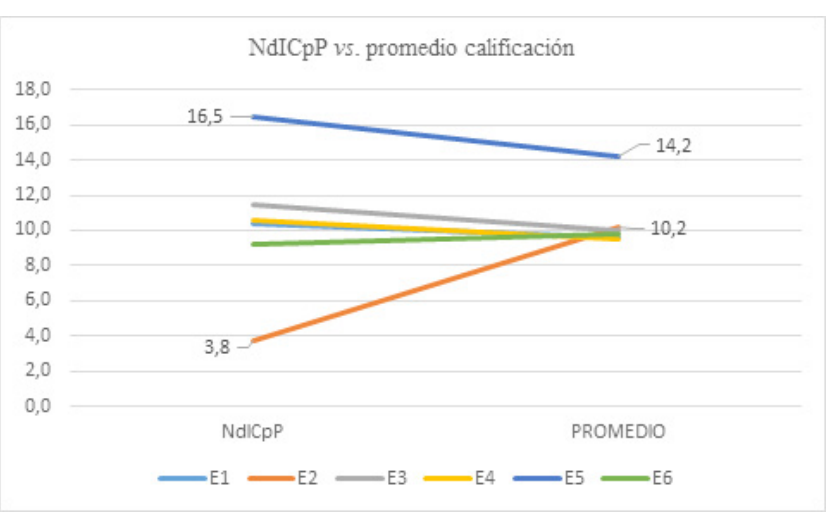

Figura 4. Contraste entre los niveles de indagación alcanzado por los equipos de laboratorio y sus respectivos promedios en la calificación del aprendizaje del postest.

Fuente: elaboración propia.

De la tabla 8, y más explícitamente de la figura 4 , se puede notar que el equipo que mejor desempeño tuvo en el NdlcpP, el E5, también logró un mejor promedio en la calificación de sus integrantes $(14,2)$. Con una tendencia similar, los equipos E1, E3, E4 y E6 obtuvieron desempeños de indagación parecidos, lo cual correspondió con los promedios de calificación que parecen converger en la figura. Sin embargo, E2 presentó un comportamiento atípico frente a lo esperado, a pesar de tener un desempeño muy bajo en la indagación $(3,8)$, superó en promedio $(10,4)$ al resto de grupos.

\section{Conclusiones}

La indagación científica abordada desde una práctica de laboratorio tipo investigación que parte de una situación problema representa en muchos casos una alternativa que logra una apropiación conceptual mucho más profunda que los métodos de enseñanza más tradicionales como el de transmisión-recepción. En este estudio se equipararon las cargas de los modelos, pues ambas metodologías contaron con prácticas de laboratorio, el segundo modelo con la práctica tipo "receta".

Aunque no es viable aplicar un solo modelo de enseñanza de las ciencias en la escuela, para este 
caso en particular, en el que se buscó el aprendizaje de la densidad como una propiedad intensiva de la materia, resultó ser más eficiente el modelo de investigación basado en competencias propias de la indagación científica. Si tenemos en cuenta que la densidad es un concepto que, aunque aparece en los libros de texto como un simple cociente entre la masa y el volumen, "los estudiantes relacionan la densidad con una de las variables (masa o volumen) y no con la relación entre ellas" (Raviolo, Moscato y Schnersch, 2005, p.90). La presencia de estas y otras dificultades presentadas en la comprensión de este concepto abstracto es precisamente la razón que hace aprovechable la indagación en búsqueda del aprendizaje, más aún cuando hay experimentación en la que existe contrastación de lo investigado y planteado en la hipótesis, a través de un experimento diseñado en equipo y ajustado en una socialización por la comunidad científica.

Al respecto de las categorías de indagación puestas en escena, y que fueron valoradas por un instrumento que se nombró como $\mathrm{NdICpP}$, se corrobora una vez más que el diseño de experimentos no es el fuerte de los estudiantes. De igual modo, el análisis de datos y obtención de conclusiones resultó en este estudio la de menor desempeño por los equipos de trabajo, quizás por la dificultad de hacer un análisis holístico desde el planteamiento del problema y la formulación de la hipótesis hasta la solución del mismo después de obtener los datos del experimento.

Sin embargo, el desempeño de los equipos de trabajo en la formulación de hipótesis resultó ser satisfactorio para este caso, contrario a lo que reportan las últimas investigaciones. El éxito en la formulación de la hipótesis parece obedecer, por una parte, a la adecuación de materiales de consulta que guardaron estrecha relación con el fenómeno y, por otra, al andamiaje conceptual prestado por el profesor durante las sesiones de trabajo. A esto hay que añadir que las experiencias en actividades de indagación han tenido un mayor efecto en el aprendizaje cuando son dirigidas por los profesores, en contraste con las que son desarrolladas por los estudiantes con toda autonomía (Furtak, Seidel y Heidy, 2012).

Todo lo anterior lleva a pensar que la formulación de hipótesis representa un ejercicio clave en la comprensión del concepto, pues hasta el equipo E2, de más bajo puntaje en el $\operatorname{NdICpP}(3,5$ de 25) pero con un desempeño regular en la formulación de hipótesis $(3,0$ de 5,0), logró buen promedio de calificación de sus integrantes $(10,4)$, inclusive mejor que el resto de equipos, excepto el (E5) cuyo promedio de calificaciones fue el más alto $(14,2)$ y que fue coherente con el puntaje del NdICpP $(16,5)$.

\section{Referencias}

Caama-o, A. (2003). Los trabajos prácticos en ciencias. En Jiménez Aleixandre, M.P., Caama-o, A., O-orbe, A., Pedrinaci, E. y de Pro, A., Ense-ar en ciencias, (pp. 95-118). Barcelona: Graó.

Couso Lagarón, D. (2014). De la moda de "aprender indagando" a la indagación para modelizar: una reflexión crítica. Conferencia plenaria inaugural en XXVI Encuentros de Didáctica de las Ciencias Experimentales. Barcelona.

Crujeiras Pérez, B. y Jiménez Aleixandre, M.P. (2015). Desafíos planteados por las actividades abiertas de indagación en el laboratorio: articulación de conocimientos teóricos y prácticos en las prácticas científicas. Ense-anza de las Ciencias, 33(1), 63-84. https://doi. org/10.5565/rev/ensciencias.1469

Ferrés Gurt, C., Marbá Tallada, A. y Neus, S.P. (2015). Trabajos de indagación de los alumnos: instrumentos de evaluación e identificación de dificultades. Eureka sobre Ense-anza y Divulgación de las Ciencias, 12(1),22-37. https://doi.org/10.25267/Rev_Eureka ensen divulg cienc.2015.v12.i1.03

Furtak, E.M., Seidel, T. y Heidy, I. (2012). Experimental and Quasi-Experimental 
Studies of Inquiry-Based Science Teaching: A Meta-Analysis. Review of Educational Research, 82(3), 300-329. https://doi. org/10.3102/0034654312457206

González Rodríguez, L. y Crujeiras Pérez, B. (2016). Aprendizaje de las reacciones químicas a través de actividades de indagación en el laboratorio sobre cuestiones de la vida cotidiana. Ense-anzas de las Ciencias, 34(3), 143-160. https://doi.org/10.5565/rev/ensciencias.2018

Hernández Sampieri, R. y Fernández Cogollo, C. (2010). Metodología de la investigación. México D.F.: Mc Graw Hill.

Lederman, N. G., Lederman, J. S., and Antink, A. (2013). Nature of science and scientific inquiry as contexts for the learning of science and achievement of scientific literacy. International Journal of Education in Mathematics, Science and Technology, 1(3).

Martin Hasen, L. (2002). Defining Inquiry: Exploring the many types of inquiry in the science classroom. The Science Teacher, 69(2), 34-37.
Raviolo, A., Moscato, M. y Schnersch, A. (2005). Ense-anza del concepto de densidad a través de un modelo analógico. Revista de la Ense-anza de la Física, 18(2), 93-103.

Romero-Ariza, M. (2017). El aprendizaje por indagación: ¿existen suficientes evidencias sobre sus beneficios en la ense-anza de las ciencias? Revista Eureka sobre ense-anza y divulgación de las ciencias, 14(2), 286-299.

Ruiz Bolívar, C. (2008). Confiabilidad (pp. 1-14). Programa Interinstitucional Doctorado en Educación. Universidad Pedagógica Experimental Libertador, Venezuela. Recuperado de: http://200.11.208.195/blogRedDocente/alexisduran/wp-content/ uploads/2015/11/CONFIABILIDAD.pdf

Sweller, J. y Clark, R. (2006). Why Minimal Guidance During Instruction Does Not Work: An Analysis of the Failure of Constructivist, Discovery, Problem-Based, Experiential, and Inquiry-Based Teaching. Educational Psychologist, 41(2), 75-86. https://doi.org/10.1207/ s15326985ep4102_ 1 


\section{Anexos}

Instrumento para caracterizar el nivel de indagación de los equipos de laboratorio en el grupo experimental y la situación problema del que parte la indagación científica.

\section{Anexo A. Nivel de indagación científica para principiantes (NdICpP).}

\begin{tabular}{|c|c|c|}
\hline \multirow[t]{3}{*}{ Categoría } & Rúbrica & Valoración \\
\hline & No plantean hipótesis o plantean hipótesis sin sentido & 0 \\
\hline & Plantean hipótesis sin relación con el problema de investigación & 1 \\
\hline \multirow{4}{*}{$\begin{array}{l}\text { A. Formulación de } \\
\text { hipótesis }\end{array}$} & Plantean hipótesis ambiguas o con errores de lógica o mal formuladas o solo emiten predicciones & 2 \\
\hline & Formulan hipótesis en forma de deducción y que encajan con el problema de investigación & 3 \\
\hline & $\begin{array}{l}\text { Formulan hipótesis en forma de deducción y que encajan con el problema de investigación y con el } \\
\text { esquema si pensamos que... entonces si... observaremos que... }\end{array}$ & 4 \\
\hline & No proponen diseño experimental & 0 \\
\hline \multirow{5}{*}{$\begin{array}{l}\text { B. Diseño } \\
\text { experimental }\end{array}$} & El diseño experimental no permite comprobar la hipótesis & 1 \\
\hline & $\begin{array}{l}\text { El diseño experimental permite comprobar la hipótesis, pero está mal organizado y requiere ajustar el } \\
\text { procedimiento }\end{array}$ & 2 \\
\hline & El diseño experimental permite comprobar la hipótesis y el procedimiento está listo para su ejecución & 3 \\
\hline & No identifican las variables implicadas en el diseño experimental & 0 \\
\hline & Proponen variables que no guardan relación con el experimento & 1 \\
\hline \multirow[t]{6}{*}{$\begin{array}{l}\text { C. Identificación } \\
\text { de variables }\end{array}$} & Identifican las variables implicadas en el experimento, pero no discriminan entre la VD y la VI & 2 \\
\hline & Identifican la VD y la VI implicadas en el experimento, pero no explican claramente la relación entre ellas & 3 \\
\hline & Identifican la VD y VI implicadas en el experimento y explican la relación entre ellas & 4 \\
\hline & No reportan datos del experimento & 0 \\
\hline & $\begin{array}{l}\text { Datos inexactos que muestran una inadecuada ejecución de los procedimientos, tratamiento } \\
\text { inadecuado de los mismos y sin gráficos }\end{array}$ & 1 \\
\hline & $\begin{array}{l}\text { Datos inexactos que muestran una inadecuada ejecución de los procedimientos, con un mal } \\
\text { tratamiento matemático, pero bien graficados }\end{array}$ & 2 \\
\hline \multirow{7}{*}{$\begin{array}{l}\text { D. Recogida y } \\
\text { procesamiento de } \\
\text { datos }\end{array}$} & $\begin{array}{l}\text { Datos inexactos que muestran una inadecuada ejecución de los procedimientos, con un buen } \\
\text { tratamiento matemático, pero mal graficados }\end{array}$ & 3 \\
\hline & $\begin{array}{l}\text { Datos inexactos que muestran una inadecuada ejecución de los procedimientos, pero con un buen } \\
\text { tratamiento matemático y bien graficados }\end{array}$ & 4 \\
\hline & $\begin{array}{l}\text { Datos exactos que muestran una adecuada ejecución de los procedimientos, pero con un mal } \\
\text { tratamiento matemático y } \underline{\text { mal graficados }}\end{array}$ & 5 \\
\hline & $\begin{array}{l}\text { Datos exactos que muestran una adecuada ejecución de los procedimientos, con un mal tratamiento } \\
\text { matemático, pero bien graficados }\end{array}$ & 6 \\
\hline & $\begin{array}{l}\text { Datos exactos que muestran una adecuada ejecución de los procedimientos, buen tratamiento } \\
\text { matemático y mal graficados }\end{array}$ & 7 \\
\hline & $\begin{array}{l}\text { Datos exactos que muestran una adecuada ejecución de los procedimientos, con un buen tratamiento } \\
\text { matemático y bien graficados. }\end{array}$ & 8 \\
\hline & Sin análisis de datos & 0 \\
\hline \multirow{3}{*}{$\begin{array}{l}\text { E. Análisis de da- } \\
\text { tos y obtención de } \\
\text { conclusiones }\end{array}$} & Análisis deficiente y conclusiones no fundamentadas en los datos, ni en elementos teóricos & 1 \\
\hline & Análisis de datos bien fundamentado, pero solo se basa en los datos empíricos o en elementos teóricos & 2 \\
\hline & Análisis de datos bien fundamentado y basado en datos empíricos y en fundamentos teóricos & 3 \\
\hline
\end{tabular}

Nota: para determinar el aporte de cada rúbrica a una categoría, por ejemplo, el $\mathrm{D}=$ Recogida y procesamiento de los datos, que cuenta con 9 rúbricas $(n=9)$, la puntuación máxima que ofrece esta es de 8 o $(n-1)$ ya que cero (0) está incluido dentro de los desempeños. Entonces el aporte de cada rúbrica para esta categoría se calcula así: 5/(9-1) = 0,625. 
Anexo B. El problema o situación de investigación: ¿ tienen igual o diferente masa los mismos volúmenes de agua, glicerina y etanol?

Adrián se encontraba en el acostumbrado descanso de la jornada escolar. Entonces, escuchó a un grupo de estudiantes que discutía lo siguiente: ¿cuál tiene mayor masa: un kilogramo de hierro o uno de algodón? Algunos respondieron apresuradamente, "un kilo de hierro", en seguida, y con confianza, otros afirmaron que el algodón; aparentemente, no porque tuvieran clara la razón, sino más bien porque creían que la respuesta no era tan obvia. El compañero que lanzó la pregunta sonrió mientras daba la respuesta "ambos tienen la misma masa, es un kilogramo de cada material". La discusión de mis compañeros de escuela me llevó a manifestarle a mi maestro de química la situación, y preguntarle acerca de cuál es la explicación científica que respondiera a la percepción de pesado y ligero que tenemos de los distintos materiales. El profe me respondió emocionado, ¡La clave está en la densidad!, no es el peso, no es la masa ni el volumen, aunque guarden relación con ello. Llevemos el interrogante a la clase de química y compartámoslo con tus compañeros, e indaguemos sobre esta propiedad tan interesante de la materia. Usemos otra pregunta y otros materiales ¿Habrá la misma masa de agua, glicerina y gasolina en los mismos volúmenes de cada sustancia? ¿Cuántos gramos de cada sustancia hay en un volumen de $1 \mathrm{ml}$ ? ¿A qué propiedad microscópica de la materia se les puede atribuir el hecho de que exista una masa constante (o de partículas) en un volumen de referencia $\left(1 \mathrm{~mL}, 1 \mathrm{~L}\right.$ ó $\left.1 \mathrm{~m}^{3}\right)$ para cada sustancia y/o material a una determinada temperatura? 